\title{
Proactive strategies for regorafenib in metastatic colorectal cancer: implications for optimal patient management
}

This article was published in the following Dove Press journal:

Cancer Management and Research

4 March 2014

Number of times this article has been viewed

\author{
Gazala Khan' \\ Rebecca A Moss ${ }^{2}$ \\ Fadi Braiteh ${ }^{3,4}$ \\ Marc Saltzman ${ }^{5}$ \\ 'Department of Hematology and \\ Oncology, Henry Ford Hospital, \\ Detroit, MI, USA; ${ }^{2}$ Rutgers Cancer \\ Institute of New Jersey, Rutgers, The \\ State University of New Jersey, New \\ Brunswick, NJ, USA; ${ }^{2}$ US Oncology \\ Research, Las Vegas, NV, USA; \\ ${ }^{4}$ Comprehensive Cancer Centers \\ of Nevada, Las Vegas, NV, USA; \\ ${ }^{5}$ Innovative Medical Research of South \\ Florida, Inc, Aventura, FL, USA
}

\begin{abstract}
Regorafenib is a broad-spectrum oral multikinase inhibitor that targets several angiogenic, oncogenic, and stromal receptor tyrosine kinases that support the tumor microenvironment. Results from the pivotal Phase III Patients with Metastatic Colorectal Cancer Treated with Regorafenib or Placebo After Failure of Standard Therapy (CORRECT) trial showed that the addition of regorafenib to best supportive care resulted in a significant improvement in median overall survival and progression-free survival compared with placebo plus best supportive care in patients with metastatic colorectal cancer (mCRC) following all available approved therapies. Thus, regorafenib is the first oral multikinase inhibitor indicated for mCRC; it currently has approval in the USA, EU, Japan, Canada, and Singapore for the treatment of $\mathrm{mCRC}$ patients who have been previously treated with fluoropyrimidine-, oxaliplatin-, and irinotecan-based chemotherapy, an anti-vascular endothelial growth factor therapy, and, if the tumor is $K R A S$ wild-type, an anti-epidermal growth factor receptor therapy. In this review, we highlight regorafenib's mechanism of action, present key efficacy data from the CORRECT trial, and discuss how to proactively manage common adverse events (eg, hand-foot skin reaction, hypertension, oral mucositis, diarrhea, and fatigue) experienced by patients receiving regorafenib. Increased awareness of potential adverse events associated with regorafenib and the implementation of proactive strategies to prevent, monitor, and manage these events early in the course of treatment will be instrumental in ensuring optimal patient management and continuation of regorafenib therapy.
\end{abstract}

Keywords: CORRECT trial, adverse-event management, multikinase inhibitor

\section{Introduction}

Recent advances in the understanding of colon cancer biology have led to a new era in the treatment of metastatic colorectal cancer (mCRC). ${ }^{1,2}$ The development of biologics to specifically target variable steps in carcinogenesis and metastasis have been incorporated into first- and second-line mCRC treatment strategies, broadening the spectrum of therapies for this disease. ${ }^{3}$ Standard treatments include cytotoxic regimens with fluoropyrimidines, oxaliplatin, or irinotecan used in combination or sequentially plus monoclonal antibodies targeting vascular endothelial growth factor (VEGF) or epidermal growth factor receptor (EGFR). ${ }^{4}$ Chemotherapy combinations with bevacizumab that specially target VEGF-A or combinations with cetuximab (an EGFR inhibitor monoclonal antibody) for patients with a $K R A S$ wild-type tumor are now standard first-line treatment options. ${ }^{4}$ For second-line therapy, combinations of other cytotoxic agents and biologics, with continued use of anti-angiogenic agents, are recommended. Upon further disease progression, other agents or combinations
Correspondence: Gazala Khan Henry Ford Hospital, 2799 West Grand Boulevard, Pallister Place

T-139, Detroit, MI, 48202, USA

$\mathrm{Tel}+13139161784$

Fax + I 313916 I266

Email gkhanI@hfhs.org 
can be used as a third-line therapy. ${ }^{4}$ Afterwards, patients have limited options other than clinical trials and exclusive supportive care. ${ }^{4}$ Thus, there is an urgent unmet clinical need for new treatment options for these patients, many of whom maintain a highly functional status.

Regorafenib is an oral multikinase inhibitor (MKI) that selectively targets three key pathologic processes implicated in cancer formation - oncogenesis, angiogenesis, and the tumor microenvironment (Figure 1). ${ }^{5,6}$ To this end, regorafenib inhibits key signaling proteins involved in oncogenesis, such as BRAF and RAF1, as well as stromal kinases that support the tumor microenvironment, such as platelet-derived growth factor receptor (PDGFR)- $\beta$ and fibroblast growth factor receptor. ${ }^{5}$ Regorafenib also suppresses receptor tyrosine kinases involved in angiogenesis, including the VEGF receptor (VEGFR)-1, -2, and -3, and a key regulator of angiogenesis, tyrosine kinase with immunoglobulin and epidermal growth factor homology domain 2 (TIE-2). ${ }^{5}$ Notably, the synergistic inhibition of both VEGFR-2 and TIE-2 leads to a greater reduction of angiogenesis than the inhibition of VEGF alone. ${ }^{5,6}$ Combined, the comprehensive inhibition of kinases involved in each of these processes is thought to deactivate colorectal tumor cells at several levels.

A Phase I, first-in-human, dose-escalation study was conducted to evaluate the safety, tolerability, and pharmacokinetics of regorafenib monotherapy in patients with advanced solid tumors. ${ }^{7}$ The study showed regorafenib to have an acceptable safety profile, similar to that of other MKIs in this drug class. The recommended dose of regorafenib for future studies was determined to be $160 \mathrm{mg}$ for 21 days, followed by 7 days off treatment (in repeating 28-day cycles). Additionally, this trial provided preliminary evidence of regorafenib's antitumor activity in heavily pretreated patients with advanced solid tumors. Therefore, an expansion cohort of patients with refractory $\mathrm{mCRC}$ was included in the extension phase of the study. ${ }^{8}$ In the expansion study, regorafenib was generally well tolerated and demonstrated antitumor activity. For example, $70 \%$ of patients with mCRC achieved stable disease while on regorafenib and $4 \%$ had a partial response as determined by Response Evaluation Criteria in

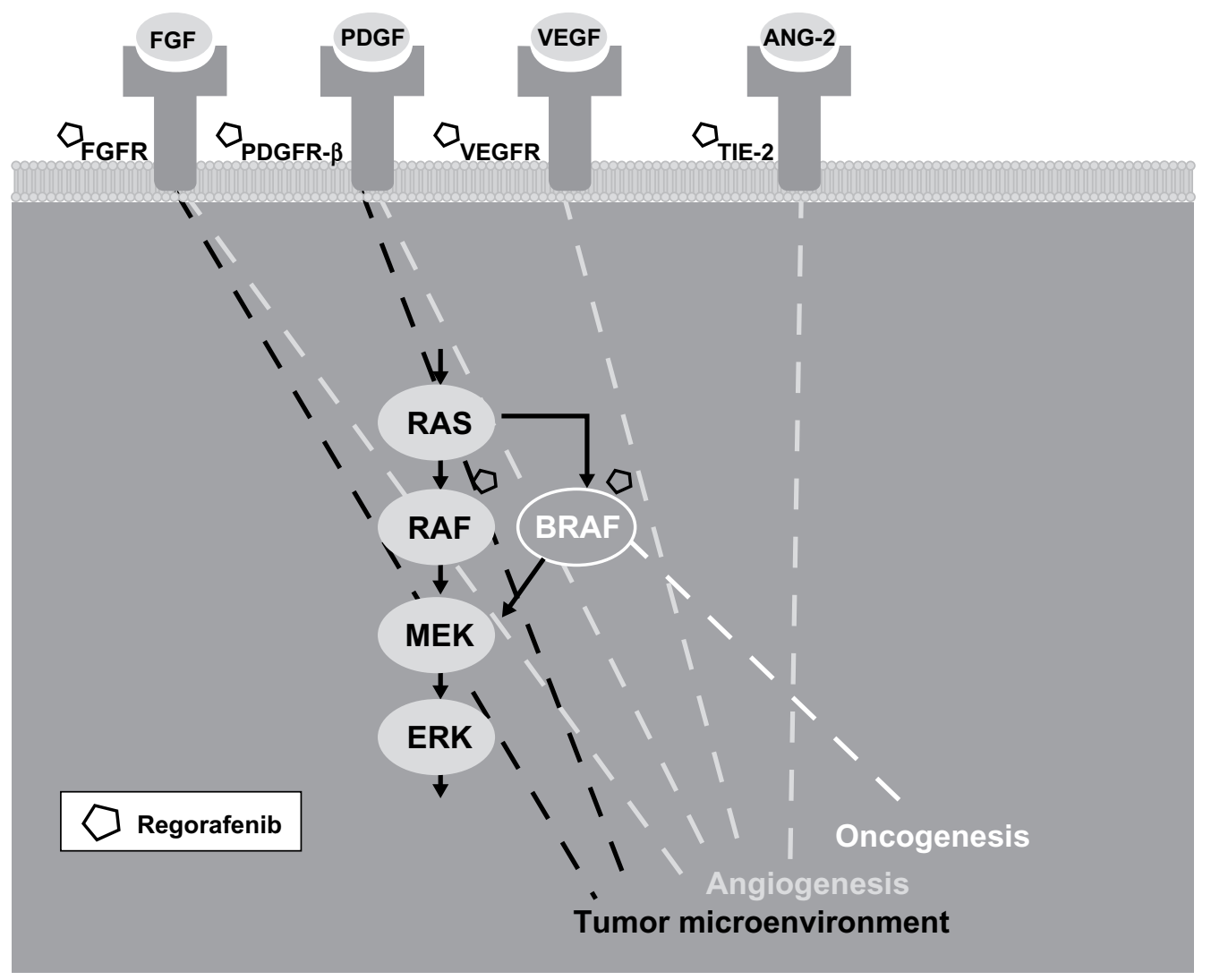

Figure I Regorafenib inhibits oncogenesis, angiogenesis, and the tumor microenvironment in metastatic colorectal cancer. Regorafenib is a multikinase inhibitor that targets several receptor tyrosine kinases (eg, fibroblast growth factor receptor [FGFR], platelet-derived growth factor receptor- $\beta$ [PDGFR- $\beta$ ], vascular endothelial growth factor receptor [VEGFR], and tyrosine kinase with immunoglobulin and epidermal growth factor homology domain [TIE2]) that regulate angiogenesis and the tumor microenvironment in colorectal tumor cells. Regorafenib also inhibits oncogenesis, in part, by selectively targeting the intracellular kinases BRAF and RAFI, an activator of downstream mitogen-activated protein kinases (MEK and ERK) that promote cell proliferation.

Abbreviation: ANG-2, angiopoietin-2. 
Solid Tumors (RECIST) version 1.1. ${ }^{8,9}$ According to RECIST, "stable disease" is defined as neither sufficient shrinkage to qualify for partial response nor sufficient increase to qualify for progressive disease, taking as reference the smallest sum diameters while on study. "Partial response" is defined as at least a $30 \%$ decrease in the sum of the diameters of target lesions, taking as reference the baseline sum diameters. ${ }^{9}$

The CORRECT (Patients with Metastatic Colorectal Cancer Treated with Regorafenib or Placebo After Failure of Standard Therapy) trial was a Phase III randomized, double-blind clinical trial evaluating the efficacy and safety of regorafenib in patients with $\mathrm{mCRC}$ after disease progression during or within 3 months of completing all standard treatments. ${ }^{10,11}$ The early findings from the CORRECT trial show that regorafenib provided statistically significant benefits in overall survival (OS) and progression-free survival (PFS) meeting its prespecified stopping criteria for efficacy. Regorafenib is now approved by the US Food and Drug Administration and several other regulatory agencies for the treatment of mCRC. ${ }^{12}$ The most recent version of the National Comprehensive Cancer Network's (NCCN) guidelines recommends regorafenib after progression on fluoropyrimidine-, oxaliplatin-, and irinotecan-based chemotherapy, an anti-VEGF therapy, and, if the tumor is $K R A S$ wild-type, an anti-EGFR therapy. ${ }^{4}$ For example, in patients with $K R A S$-mutant tumors, regorafenib may be used following a second progression on FOLFOX (leucovorin [folinic acid], 5-fluorouracil, and oxaliplatin) or FOLFIRI (folinic acid, 5-fluorouracil, and irinotecan), when administered with bevacizumab. ${ }^{4}$ In patients with $K R A S$-wild-type tumors, regorafenib may be used following a second progression on FOLFOX or FOLFIRI, as long as bevacizumab and cetuximab were used in either first- or second-line. ${ }^{4}$ In accordance with NCCN guidelines, clinicians may also consider enrolling patients who maintain adequate performance status and end-organ function into clinical trials, including Phase I trials, after at least two progressions on standard treatments. ${ }^{4}$

The following review summarizes the clinical outcomes of the CORRECT trial and discusses the clinical use of regorafenib, including dosing, adverse effects, and the management of toxicity.

\section{Phase III CORRECT study Study design and patients}

CORRECT was an international, randomized, multicenter, double-blind, placebo-controlled Phase III clinical trial conducted to determine the efficacy and safety of regorafenib in patients with $\mathrm{mCRC}$ who had progressed during or within 3 months of previous standard-of-care therapy (ie, a fluoropyrimidine, oxaliplatin, irinotecan, bevacizumab, and cetuximab or panitumumab if the tumor was $K R A S$ wild-type). ${ }^{11}$ The primary endpoint was OS. The secondary endpoints included PFS, objective tumor response rate (assessed every 8 weeks), disease control rate, safety, and health-related quality-of-life analysis. Importantly, patients enrolled in the CORRECT trial represented a heavily pretreated population, in that nearly half of each treatment group had received $\geq 4$ prior lines of systemic anticancer therapy for metastatic disease, and all patients had been previously treated with bevacizumab.

Patients $(\mathrm{N}=760)$ were randomized in a $2: 1$ ratio to receive oral regorafenib $160 \mathrm{mg}(\mathrm{N}=505)$ or placebo $(\mathrm{N}=255)$, according to a dosing schedule of 3 weeks on and 1 week off, until disease progression or unacceptable toxicity. Patients were stratified by prior VEGF therapy, time from diagnosis ( $\geq 18$ or $<18$ months), and geographical region. No crossover between treatment groups was allowed. The study used a $90 \%$ power to detect a $33.3 \%$ increase in median OS (the primary endpoint), assuming a 4.5-month median OS for the placebo group (hazard ratio $[\mathrm{HR}]=0.75$ ).

\section{Efficacy results}

\section{Survival analysis}

The study met its prespecified stopping criteria for efficacy at the second preplanned interim analysis of OS (after 432 events). The addition of regorafenib to best supportive care resulted in a median OS of 6.4 months (interquartile range [IQR]: 3.6-11.8) compared with a median of 5 months (IQR: 2.8-10.4) for those in the placebo group ( $\mathrm{HR}=0.77$ [95\% confidence interval $\{\mathrm{CI}\}: 0.64-0.94] ; P=0.0052$ ) (Figure 2A). ${ }^{11}$ At the final analysis of OS, the benefit of regorafenib was confirmed. Unlike median values, the HR reflects the effect of regorafenib for the entire study period. ${ }^{13}$ In this trial, the HR for OS at final analysis was $0.79(95 \%$ CI: $0.66-0.94 ; P=0.0038$ ) for regorafenib versus placebo, resulting in a $21 \%$ reduced risk of death with regorafenib and best supportive care. ${ }^{14}$ The median OS rate at 6 months was $52.2 \%$ in the regorafenib group and $43.1 \%$ in the placebo group; at 12 months, the median OS rate was $24.1 \%$ and $17.0 \%{ }^{14}$ In an unplanned subgroup analysis for OS, regorafenib showed apparent survival benefit across all subgroups, except the subgroup with primary disease in the colon and the rectum (HR =1.09). ${ }^{11}$ However, it is worth mentioning that this was a small subgroup and the analysis was not powered for statistical significance. ${ }^{11}$ Also, while 

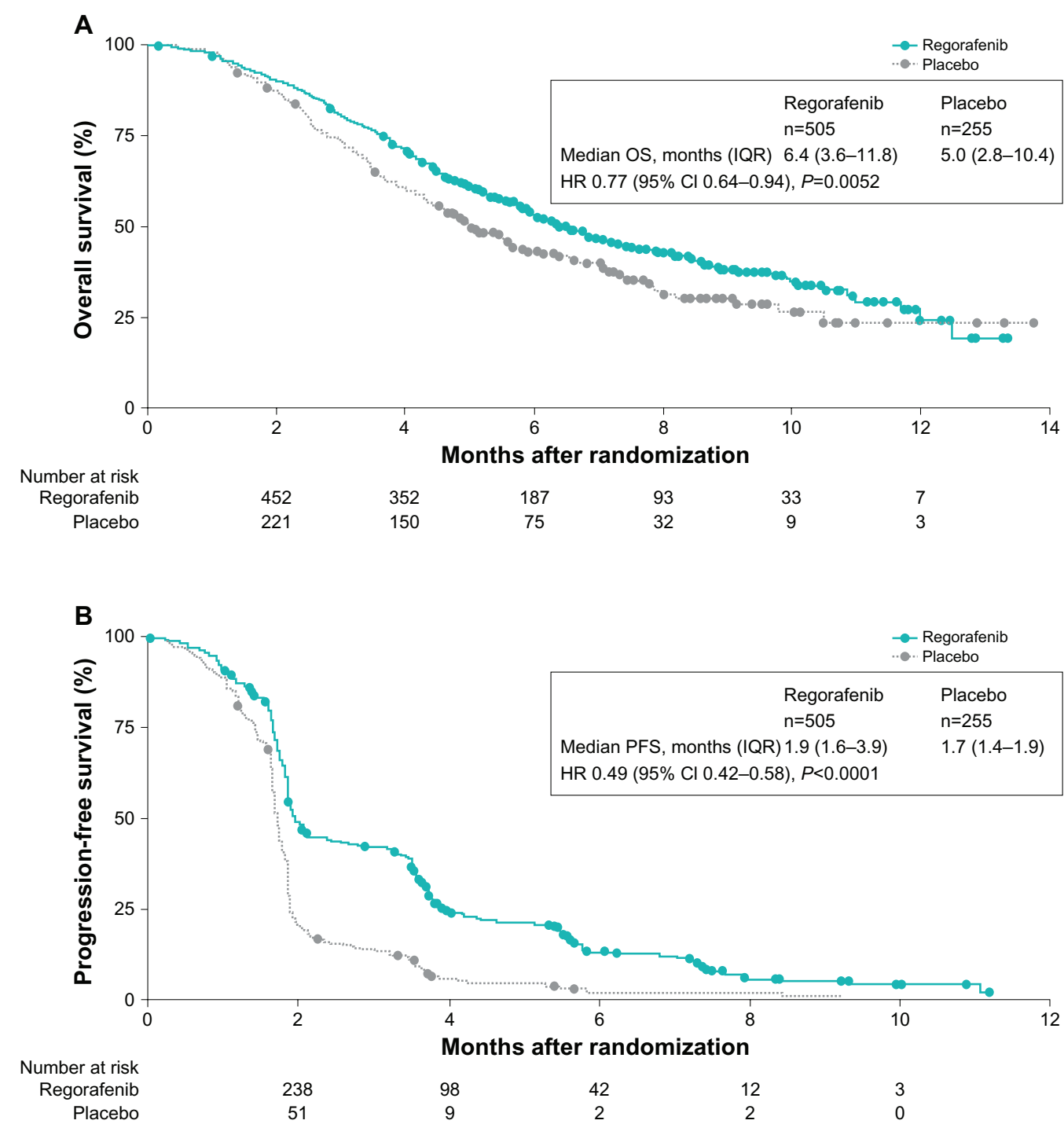

Figure 2 Kaplan-Meier curves for overall survival (A) and progression-free survival (B) in the study population.

Notes: The hazard ratio (HR) for overall survival at final analysis was 0.79 (95\% Cl: 0.66-0.94; $P=0.0038$ ). Reprinted from The Lancet, 38I, Grothey et al, Regorafenib monotherapy for previously treated metastatic colorectal cancer (CORRECT): an international, multicentre, randomised, placebo-controlled, phase 3 trial, 303-312, Copyright (C) 2013, with permission from Elsevier."I

Abbreviations: $\mathrm{Cl}$, confidence interval; IQR, interquartile range; PFS, progression-free survival.

regorafenib appeared to have a greater OS benefit in colon cancer $(\mathrm{HR}=0.70)$ compared with rectal cancer $(\mathrm{HR}=0.95)$, rectal cancer patients who received placebo had more therapies following progression than the overall population ( $36 \%$ vs $30 \%) .{ }^{11}$ In contrast, rectal cancer patients who received regorafenib had fewer therapies following progression than the overall population ( $23 \%$ vs $26 \%$ ). ${ }^{11}$ Overall, regorafenib appeared to provide a survival benefit over placebo regardless of whether patients had longer or shorter time from first diagnosis of $\mathrm{mCRC}$, prior anticancer treatment, and more or less than three lines of therapy. ${ }^{11}$ With regard to KRAS status, subgroup analysis showed regorafenib mediated a survival benefit over placebo in the $K R A S$-wild-type $(\mathrm{HR}=0.65)$ and KRAS-mutant $(\mathrm{HR}=0.87)$ subgroups. ${ }^{11}$
In addition, regorafenib statistically improved PFS from 1.7 months in the placebo arm to 1.9 months in the regorafenib $\operatorname{arm}(\mathrm{HR}=0.49 ; 95 \%$ CI $0.42-0.58 ; P<0.0001)\left(\right.$ Figure 2B) ${ }^{11}$ This was maintained during the course of the study: the median PFS rate was longer in the regorafenib arm at 3, 6, and 9 months post-randomization. In contrast to OS subgroup analysis, patients with both colon and rectal cancers showed longer PFS over placebo, suggesting that regorafenib has clinical benefit in both tumor types. Moreover, the benefit in OS and PFS was independent of treatment history and KRAS status. ${ }^{11}$

\section{Objective responses}

Patients in the CORRECT trial were also evaluated for objective tumor responses according to RECIST criteria. 
As expected, no patients had a complete response; however partial responses were achieved by five (1\%) patients in the regorafenib group and one $(0.4 \%)$ patient in the placebo group, yielding an objective response rate of $1 \%$ and $0.4 \%$ respectively $(P=0.19) .{ }^{11}$ However, the disease control rate (partial response + stable disease assessed at least 6 weeks after randomization) was $41 \%$ for the patients treated with regorafenib and $15 \%$ for those treated with placebo $(P<0.0001)$, indicating that regorafenib provides clinical benefit, in part by inducing stable disease in mCRC patients. ${ }^{11}$

In a retrospective analysis, a subset of patients enrolled at one center in the CORRECT trial experienced a decrease in radiological tumor density as well as cavitation of lung metastases early on during the course of treatment ( 8 weeks post-baseline evaluation). ${ }^{15}$ While the investigators only analyzed data from 22 patients receiving regorafenib (15 patients receiving placebo), they found a significant association between patients who experienced tumor shrinkage, cavitation of lung metastases, or low tumor density at baseline and favorable clinical outcome (in terms of OS and PFS). While these data are preliminary, they suggest that early imaging analysis may be predictive of clinical outcome in patients treated with regorafenib. This type of analysis is beneficial and worthy of further evaluation in more patients.

\section{Safety}

Regorafenib was generally well tolerated. The most common adverse events (AEs) of any grade that occurred in $\geq 10 \%$ of patients in the CORRECT study included fatigue, decreased appetite and food intake, hand-foot skin reaction (HFSR), also known as palmar-plantar erythrodysesthesia or PPE, diarrhea, mucositis, weight loss, infection, and hypertension (Table 1). ${ }^{12}$ In addition, increased levels of alanine aminotransferase (ALT), aspartate aminotransferase (AST), and hyperbilirubinemia were observed in some patients (Table 2). The majority of the elevations in AST, ALT, and bilirubin were grade 1 and 2 AEs, and clinically nonsignificant. ${ }^{11}$ Notably, the discontinuation rate due to drug-related AEs was low for regorafenib-treated patients $(8.2 \%$ vs $1.2 \%$ for placebo-treated patients), suggesting that many AEs can be generally managed over the treatment course.

Interestingly, most AEs occurred early in the treatment course, during cycles 1 and 2, and their incidence decreased over subsequent cycles of therapy (Figure 3). ${ }^{16}$ Further, the dosing of regorafenib remained relatively stable after three cycles of treatment, and there was no evidence of cumulative toxicity. ${ }^{12,17}$ These data suggest that the incidence of drug-related AEs decreases over time, and that proactive AE management early on may help to decrease the incidence or severity of those AEs that occur during the course of treatment, thus enabling better treatment compliance and adherence to therapy.

\section{AE management}

Clinical experience has shown that certain side effects associated with regorafenib, most notably HFSR, skin rash, and hypertension, are also associated with other MKIs. ${ }^{18}$

Table I Incidence of adverse events ( $\geq 10 \%)$ reported in patients treated with regorafenib in the CORRECT study and reported more commonly than in patients receiving placebo

\begin{tabular}{|c|c|c|c|c|}
\hline \multirow[t]{2}{*}{ Adverse event } & \multicolumn{2}{|c|}{ Regorafenib $(\mathrm{N}=500)$} & \multicolumn{2}{|c|}{ Placebo $(\mathrm{N}=\mathbf{2 5 3})$} \\
\hline & All grades (\%) & Grade $\geq 3$ (\%) & All grades (\%) & Grade $\geq 3(\%)$ \\
\hline Asthenia/fatigue & 64 & 15 & 46 & 9 \\
\hline Decreased appetite and food intake & 47 & 5 & 28 & 4 \\
\hline HFSR/PPE & 45 & 17 & 7 & 0 \\
\hline Diarrhea & 43 & 8 & 17 & 2 \\
\hline Mucositis & 33 & 4 & 5 & 0 \\
\hline Weight loss & 32 & $<1$ & 10 & 0 \\
\hline Infection & 31 & 9 & 17 & 6 \\
\hline Hypertension & 30 & 8 & 8 & $<1$ \\
\hline Dysphonia (voice changes) & 30 & 0 & 6 & 0 \\
\hline Pain & 29 & 3 & 21 & 2 \\
\hline Fever & 28 & 2 & 15 & 0 \\
\hline Rash $^{\mathrm{a}}$ & 26 & 6 & 4 & $<1$ \\
\hline Hemorrhage & 21 & 2 & 8 & $<1$ \\
\hline Headache & 10 & $<1$ & 7 & 0 \\
\hline
\end{tabular}

Notes: aRash represents events of drug eruption, rash, erythematous rash, generalized rash, macular rash, maculo-papular rash, popular rash, and pruritic rash. Data taken from Stivarga ${ }^{\circledR}$ (regorafenib) tablets prescribing information, from Bayer HealthCare Pharmaceuticals, Inc. Copyright (C) $2013 .^{12}$

Abbreviations: HFSR, hand-foot skin reaction; PPE, palmar-plantar erythrodysesthesia. 
Table 2 Incidence of liver function test abnormalities for patients in the safety population who had liver function tests

\begin{tabular}{|c|c|c|c|c|c|c|}
\hline \multirow[t]{2}{*}{ Adverse event } & \multicolumn{3}{|c|}{ Regorafenib $(\mathrm{N}=500)$} & \multicolumn{3}{|c|}{ Placebo $(\mathrm{N}=253)$} \\
\hline & All grades (\%) & Grade 3 (\%) & Grade 4 (\%) & All grades (\%) & Grade 3 (\%) & Grade 4 (\%) \\
\hline Hyperbilirubinemia & 45 & 10 & 3 & 17 & 5 & 3 \\
\hline Increased AST & 65 & 5 & 1 & 46 & 4 & 1 \\
\hline Increased ALT & 45 & 5 & 1 & 30 & 3 & $<1$ \\
\hline
\end{tabular}

Note: Data taken from Stivarga ${ }^{\circledR}$ (regorafenib) tablets prescribing information, from Bayer HealthCare Pharmaceuticals, Inc. Copyright @ 20 I3. ${ }^{\prime 2}$

Abbreviations: ALT, alanine aminotransferase; AST, aspartate aminotransferase.

However, the pathophysiologic mechanisms underlying the development of these toxicities are not well understood. ${ }^{18}$ It has been suggested that HFSR may be an indirect effect of the combined inhibition of VEGFR and PDGFR..$^{19}$ Inhibition of these receptors could potentially interfere with vascular repair mechanisms, resulting in HFSR in high-pressure areas that are exposed to subclinical trauma. ${ }^{19}$ As for MKI-induced hypertension, it has been proposed that the increase in blood pressure may be the result of abnormalities in endothelial function due to impaired angiogenesis. ${ }^{18}$ While a more thorough understanding of the molecular mechanisms underlying these MKI-induced toxicities is needed, recommendations on how to proactively manage those AEs during the course of treatment can help optimize treatment compliance and adherence to therapy.

\section{HFSR}

In the CORRECT trial, $47 \%$ of patients treated with regorafenib experienced HFSR compared with $8 \%$ of patients treated with placebo. ${ }^{11}$ HFSR may develop soon after a patient starts therapy, usually within the first $2-4$ weeks of initiating treatment. ${ }^{19,20}$ HFSR usually manifests on skin surfaces where there is pressure or friction and is characterized by asymmetric localized hyperkeratotic lesions surrounded by erythematous regions. The affected areas become painful scaly lesions that may blister and thicken, significantly interfering with the patient's everyday activities. Management of HFSR begins with prophylactic measures that were utilized in the CORRECT study protocol and are described below. ${ }^{16}$

Proactive AE management should be implemented before skin lesions develop; this approach may lessen the severity
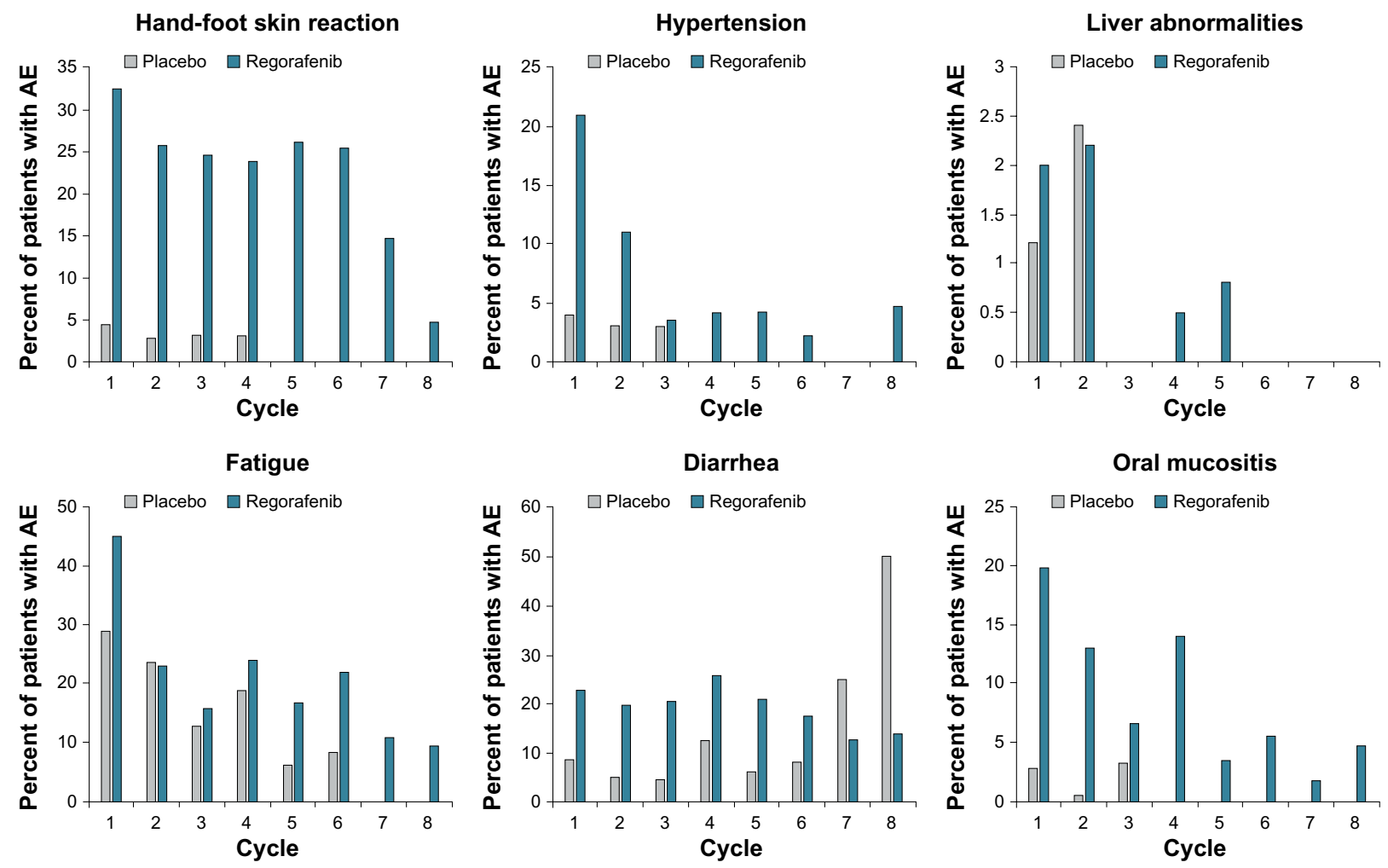

Figure 3 Time course of treatment-emergent adverse events in the CORRECT trial.

Note: Shown is the incidence of adverse events (AEs) over eight 4-week treatment cycles (one treatment cycle was 3 weeks of regorafenib I60 mg [or matching placebo] orally once daily and I week off treatment). 
and shorten the duration of the condition. ${ }^{16}$ Prior to treatment, patients should remove any calluses on their hands and feet. A pumice stone is recommended for callus or "rough spot" removal, and, when indicated, a pedicure and/or manicure may be helpful. To help prevent callus formation during treatment, patients should avoid pressure points and items that rub, pinch, or create friction. Pressure points and tender areas should be protected with cotton gloves/socks. Patients should wear well-padded, well-fitting shoes. Foot soaks with tepid water and Epsom salts and the use of insole cushions or inserts should help protect tender areas of the feet. Hands should be protected with padded gloves when engaging in activities that might irritate or damage the skin. Non-ureabased creams should be applied liberally to soften the skin of the hands and feet, while keratolytic creams, containing urea or salicylic acid $6 \%$ can be used sparingly on affected (hyperkeratotic) areas. Alpha hydroxyl acid-based creams $(5 \%-8 \%)$ can be applied liberally two times a day to gently exfoliate callused skin. ${ }^{16}$ Although the prophylactic use of urea-based cream was not part of the CORRECT trial, the prophylactic topical use of urea-based cream has been shown to help prevent and/or delay the incidence of HFSR associated with sorafenib in patients with hepatocellular carcinoma. ${ }^{21}$

Additional measures to minimize the risk of HFSR include avoiding hot water when washing, showering, and bathing, and avoiding clothing that rubs or irritates the skin. While these practical measures may be used to manage grade 1 symptoms, effective management of grade 2 and grade 3 symptoms might require dose modifications or interruptions. Dose reduction and/or temporary interruption of regorafenib, or in severe or persistent cases, discontinuation of regorafenib should be considered (Table 3 ). In the CORRECT trial; $32 \%$ of regorafenib-treated patients had symptoms of HFSR during cycle 1 but only $5 \%$ in cycle 8 (all HFSR grade 1) (Figure 3). ${ }^{16}$ The tapering incidence of HFSR over later cycles suggests that proactive AE management may be effective in reducing the frequency and severity of this condition.

\section{Hypertension}

Hypertension is a common AE thought to be associated with most MKIs and angiogenesis inhibitors and management recommendations should be adapted from the 2003 Joint National Committee on Prevention, Detection, Evaluation, and Treatment of High Blood Pressure (JNC 7) guidelines. ${ }^{22-24}$ According to the JNC 7 guidelines, "hypertension" is defined as systolic blood pressure of $\geq 140 \mathrm{mmHg}$ or diastolic blood pressure of $\geq 90 \mathrm{mmHg}$. ${ }^{24}$ Additionally, patients with systolic
Table 3 Guidelines for modifying the regorafenib dose

\begin{tabular}{|c|c|}
\hline Interrupt & $\begin{array}{l}\text { - NCI CTCAE grade } 2 \text { HFSR/PPE that is recurrent } \\
\text { or does not improve within } 7 \text { days despite dose } \\
\text { reduction; interrupt therapy for a minimum of } \\
7 \text { days for grade } 3 \text { HFSR } \\
\text { - Symptomatic grade } 2 \text { hypertension } \\
\text { - Any } \mathrm{NCl} \text { CTCAE grade } 3 \text { or } 4 \mathrm{AE}\end{array}$ \\
\hline $\begin{array}{l}\text { Reduce to } \\
120 \mathrm{mg}\end{array}$ & $\begin{array}{l}\text { - The first occurrence of grade } 2 \text { HFSR of any } \\
\text { duration } \\
\text { - After recovery of any grade } 3 \text { or } 4 \text { AE } \\
\text { - Grade } 3 \text { AST/ALT elevation; only resume if } \\
\text { the potential benefit outweighs the risk of } \\
\text { hepatotoxicity }\end{array}$ \\
\hline Reduce to & - Recurrence of grade 2 HFSR at the $120 \mathrm{mg}$ dose \\
\hline $80 \mathrm{mg}$ & $\begin{array}{l}\text { After recovery of any grade } 3 \text { or } 4 \mathrm{AE} \text { at the } \\
120 \mathrm{mg} \text { dose (except hepatotoxicity) }\end{array}$ \\
\hline $\begin{array}{l}\text { Discontinue } \\
\text { permanently }\end{array}$ & $\begin{array}{l}\text { - Failure to tolerate } 80 \mathrm{mg} \text { dose } \\
\text { - Any occurrence of AST/ALT }>20 \times \mathrm{ULN} \\
\text { - Any occurrence of AST/ALT }>3 \times \mathrm{ULN} \text { with } \\
\text { concurrent bilirubin }>2 \times \mathrm{ULN} \\
\text { - Recurrence of AST/ALT }>5 \times \mathrm{ULN} \text { despite dose } \\
\text { reduction to } 120 \mathrm{mg} \\
\text { - Any grade } 4 \text { adverse reaction; only resume if the } \\
\text { potential benefit outweighs the risks }\end{array}$ \\
\hline
\end{tabular}

Note: Recommended regorafenib dose modifications taken from Stivarga ${ }^{\otimes}$ (regorafenib) tablets prescribing information, from Bayer HealthCare Pharmaceuticals Inc. ${ }^{12}$

Abbreviations: AE, adverse event; ALT, alanine aminotransferase; $A S$, aspartate aminotransferase; CTCAE, Common Terminology Criteria for Adverse Events; HFSR, hand-foot skin reaction; NCI, National Cancer Institute; PPE, palmar-plantar erythrodysesthesia; ULN, upper limit of normal.

blood pressure of 120-139 mmHg or diastolic blood pressure of $80-89 \mathrm{mmHg}$ are considered prehypertensive and these patients may require additional blood-pressure monitoring. ${ }^{24}$ The same criteria were employed in the CORRECT trial protocol to define hypertension, as well as a $\geq 20 \mathrm{mmHg}$ increase in diastolic measurement if blood pressure was previously within the normal range. ${ }^{16}$

In the CORRECT trial, hypertension was reported in $28 \%$ of regorafenib-treated patients compared with $6 \%$ of placebotreated patients. ${ }^{11}$ In most patients, the onset of hypertension occurred during the first cycle of regorafenib treatment.

In accordance with the CORRECT trial protocol, blood pressure should be measured and adequately controlled before starting treatment, according to standard medical practice. ${ }^{16}$ After starting treatment, blood pressure should be monitored weekly for the first 6 weeks of treatment and patients should be advised to regularly perform home bloodpressure monitoring. ${ }^{25}$ Patients should also be advised to record their blood-pressure readings in a diary and notify the health care team of any elevations.

If high blood pressure develops during regorafenib treatment, there are several classes of oral antihypertensive agents that can be prescribed to manage treatment-induced 
hypertension. ${ }^{25}$ These include thiazide diuretics, beta blockers, dihydropyridine and non-dihydropyridine calcium channel antagonists, angiotensin-converting enzyme inhibitors, and angiotensin-receptor antagonists. The JNC 7 guidelines recommend using two agents when blood pressure is $>20 / 10 \mathrm{mmHg}$ above target blood-pressure range; generally one of these medications will be a thiazide diuretic. ${ }^{24}$

All of the classes of antihypertensive agents have been used to successfully control VEGF signaling pathway inhibitor-induced hypertension on an individual patient basis. ${ }^{25}$ In addition to careful blood-pressure monitoring and attentive review of the antihypertensive regimen, effective management of treatment-induced hypertension might require regorafenib dose modifications. Suggested dose modifications are highlighted in Table 3.

\section{Liver abnormalities}

Abnormalities of liver function tests (LFTs) have been observed in patients treated with MKI therapy. ${ }^{26,27}$ In the CORRECT trial, increased levels of ALT, AST, and bilirubin were observed more frequently in patients treated with regorafenib than with placebo. These elevations, however, were mainly due to grade 1 and 2 events. ${ }^{11}$ These LFT abnormalities occurred early in the course of treatment, during cycles 1 and 2 , and were readily manageable with dose modification or interruption. ${ }^{11}$ Severe LFT abnormalities or hepatic dysfunction were observed in a small number of patients. ${ }^{11}$

Therefore, it is advised that liver function be monitored prior to and during regorafenib therapy. ${ }^{16}$ The early detection of liver function abnormalities requires active patient monitoring, including LFTs at least every 2 weeks during the first 2 months of treatment. After the first 2 months, LFTs should be conducted at least monthly; however, more frequent monitoring may be required for some patients. In addition, patients with elevated LFTs should be monitored weekly until improvement to less than three times the upper limit of normal or baseline (see Table 4 for grading of LFT elevations). In some cases, a change in the regorafenib dose or an interruption in the treatment schedule may be needed (Table 5).

\section{Diarrhea}

In the CORRECT trial, 34\% of the regorafenib-treated patients reported diarrhea (mainly grade 1 and 2), compared with $8 \%$ of patients receiving placebo. ${ }^{11}$ These rates are similar to those observed with other MKIs, including sunitinib, sorafenib, and imatinib, which have been reported to cause diarrhea in approximately one-third of treated patients. ${ }^{28}$

Initial management of mild to moderate diarrhea may include dietary modifications and the use of antidiarrheal medications, such as loperamide, octreotide, and tincture of opium. ${ }^{28,29}$ Diarrhea must be treated promptly, as loss of fluids and electrolytes associated with persistent or severe diarrhea can result in life-threatening dehydration. ${ }^{29}$ Patients who experience persistent or severe diarrhea should be monitored for electrolyte abnormalities, such as low magnesium and potassium, and for bleeding hemorrhoids. Management of diarrhea should include a dietary intake of at least eight glasses of clear fluids daily and small frequent meals, with high-soluble-fiber foods (eg, bananas and rice) and avoidance of all lactose-containing products, alcohol, and high-osmolar supplements. ${ }^{29}$ Adherence to these dietary recommendations, along with the use of antidiarrheal medications, when needed, may provide adequate diarrhea control and lessen the need to reduce the dose of regorafenib or discontinue treatment.

\section{Potential comorbidities}

Fatigue is a common condition in patients with cancer that affects quality of life and interferes with usual functioning. ${ }^{30}$ The cause of cancer-related fatigue is not fully understood, but it may not be directly attributable to drug therapy. In the CORRECT trial, $47 \%$ of regorafenib-treated patients and $28 \%$ of placebo-treated patients reported fatigue. ${ }^{11}$ Initial management of fatigue begins with excluding reversible treatable common causes of fatigue, such as pain, sleep disturbance, and anemia - conditions that can be improved with medical treatment. ${ }^{30}$ Since hypothyroidism may contribute to severe fatigue, patients receiving regorafenib who experience severe fatigue should be evaluated for thyroid dysfunction. After addressing potential contributory factors,

Table 4 Management of regorafenib-related liver function abnormalities: grading of abnormalities ${ }^{\mathrm{a}}$

\begin{tabular}{llllll}
\hline Serum level on LFT & Grade I & Grade 2 & Grade 3 & Grade 4 & Grade 5 \\
\hline ALT/AST & $>$ ULN to $3 \times$ ULN & $>3$ to $5 \times$ ULN & $>5$ to $20 \times$ ULN & $>20 \times$ ULN & NA \\
Bilirubin $^{\text {b }}$ & $>$ ULN to $1.5 \times$ ULN & $>1.5$ to $3.0 \times$ ULN & $>3$ to $10 \times$ ULN & $>10 \times$ ULN & NA \\
\hline
\end{tabular}

Notes: aGrading by the National Cancer Institute Common Terminology Criteria for Adverse Events (version 4.03.20 I0); 'exception: patients with Gilbert's syndrome who develop elevated transaminases should be managed as per the outlined recommendations for the respective observed elevation of ALT and/or AST.

Abbreviations: ALT, alanine aminotransferase; AST, aspartate aminotransferase; LFT, liver function test; NA, not applicable; ULN, upper limit of normal. 
Table 5 Management of regorafenib-related liver function abnormalities: dose-modification guidelines

\begin{tabular}{|c|c|c|c|}
\hline Grade $^{a}$ & $\begin{array}{l}\text { ALT and/or AST } \\
\text { elevations }\end{array}$ & Occurrence & Recommended measures \\
\hline$\leq$ Grade 2 & $\leq 5 \times$ ULN & Any occurrence & $\begin{array}{l}\text { - Continue treatment } \\
\text { - Monitor liver function weekly until transaminases return to } \\
<3 \times \text { ULN (grade I) or baseline }\end{array}$ \\
\hline $\begin{array}{l}\geq \text { Grade } 2 \text { with concurrent } \\
\text { bilirubin }>2 \times \text { ULN }\end{array}$ & $>3 \times$ ULN & Any occurrence ${ }^{b}$ & $\begin{array}{l}\text { - Discontinue treatment permanently } \\
\text { - Monitor liver function weekly until resolution or return to baseline }\end{array}$ \\
\hline Grade 3 & $>5 \times$ to $\leq 20 \times$ ULN & First occurrence & $\begin{array}{l}\text { - Interrupt treatment } \\
\text { - Monitor transaminases weekly until levels return to }<3 \times \text { ULN or } \\
\text { baseline } \\
\text { - Patients may restart treatment if the potential risk outweighs the } \\
\text { risk of liver toxicity } \\
\text { - To restart treatment, reduce dose by I tablet }(40 \mathrm{mg}) \text { and monitor } \\
\text { liver function weekly for at least } 4 \text { weeks }\end{array}$ \\
\hline & & Recurrence & - Discontinue treatment permanently \\
\hline Grade 4 & $>20 \times U L N$ & Any occurrence & - Discontinue treatment permanently \\
\hline
\end{tabular}

Notes: aGrading by the National Cancer Institute Common Terminology Criteria for Adverse Events (version 4.03.20 I0); bexception: patients with Gilbert's syndrome who develop elevated transaminases should be managed as per the outlined recommendations for the respective observed elevation of ALT and/or AST.

Abbreviations: ALT, alanine aminotransferase; AST, aspartate aminotransferase; ULN, upper limit of normal.

patients with cancer-related fatigue may be treated with pharmacologic and non-pharmacologic interventions. ${ }^{30}$ For patients with mild fatigue, non-pharmacologic approaches such as individual counseling, stress management, or sleep therapy may be sufficient; patients with moderate to severe fatigue may benefit from pharmacologic treatment. ${ }^{30}$ Interventions may also be implemented to ensure the patient has adequate nutrition and hydration, which may help reduce fatigue. $^{31,32}$

In the CORRECT trial, asymptomatic hypophosphatemia was reported in $5 \%$ of patients receiving regorafenib compared with $<1 \%$ of patients receiving placebo. ${ }^{11}$ While the etiology of hypophosphatemia associated with MKI therapy is not known, it is a common laboratory finding in patients undergoing treatment with MKIs. ${ }^{27,33}$ Elevated lipase is another common laboratory abnormality associated with MKI therapy. ${ }^{26,27,33,34}$ Although it is not known why these effects are observed, phosphorus and lipase levels should be monitored frequently during initial cycles of treatment and throughout the course of treatment, as some patients may require dose modifications or dose interruptions at some point during therapy.

\section{Dosing}

The recommended regorafenib dose is $160 \mathrm{mg}(4 \times 40 \mathrm{mg}$ tablets daily). ${ }^{12}$ This dose should be taken orally with a low-fat meal once daily for the first 21 days of each 28 -day cycle. ${ }^{12}$ While $160 \mathrm{mg}$ is the prescribed dose according to the prescribing information, dose reduction may be necessary for proper AE management.

\section{Patient adherence}

Patient adherence to therapy can be challenging with oral anticancer agents. The complexity and duration of treatment, frequent changes in the regimen, and side effects are common barriers to adherence to a prescribed treatment regimen. Increased communication between patients and physicians can help overcome these barriers. Frequent patient visits and encouraging patients to express their concerns can help establish a sense of collaboration that may improve adherence and facilitate AE management.

\section{Ongoing studies}

Currently, regorafenib is being investigated in combination with other agents for the treatment of mCRC. One openlabel, international Phase II trial evaluated the efficacy and safety of regorafenib when given with modified FOLFOX (mFOLFOX6) as first-line treatment in patients with mCRC. ${ }^{35}$ The study showed that regorafenib, when combined with mFOLFOX6, has an acceptable safety profile. While this study reported some efficacy for regorafenib and FOLFOX, definitive conclusions cannot be made from a single arm study. Another ongoing Phase II study is the randomized, multicenter, placebo-controlled US trial of regorafenib in combination with FOLFIRI as second-line treatment in patients with mCRC..$^{36}$ This trial is designed to compare PFS between regorafenib plus FOLFIRI (Arm A) versus placebo plus FOLFIRI (Arm B) in patients with mCRC who were previosly treated with a FOLFOX regimen.

In addition, because of the high unmet need for treatment options in this patient population, a Phase IIIB, prospective, 
interventional, open-label, single-arm, multicenter study (CONSIGN) is designed to provide regorafenib to patients diagnosed with $\mathrm{mCRC}$ who have failed after standard therapy and for whom no therapy alternatives exist. ${ }^{37}$ CONSIGN provides early access in countries where regorafenib has not yet been approved, and will collect safety data for regorafenib until market access.

\section{Conclusion}

CORRECT is the first randomized placebo-controlled Phase III study of an oral MKI to have showed efficacy and safety in patients with heavily pretreated mCRC who progressed on prior standard-of-care treatments. All patients had received prior treatment with bevacizumab and fluoropyrimidine-, oxaliplatin-, and irinotecan-based chemotherapy. Patients with $K R A S$-wild-type tumors had received prior treatment with cetuximab and/or panitumumab. ${ }^{10}$ The study shows that regorafenib provides survival benefits in mCRC that has progressed after all standard therapies. The safety profile of regorafenib in CORRECT indicates that it is a generally well-tolerated treatment with manageable AEs using current clinical practice and proposed risk-management guidelines. The AEs associated with regorafenib treatment are consistent with those routinely managed by oncologists. Moreover, a comprehensive health-related quality-of-life analysis showed that there were no significant differences in patient quality of life between those individuals receiving regorafenib and those receiving placebo. ${ }^{11}$ Overall, the approval of regorafenib represents a new standard-of-care therapy for heavily pretreated patients who previously had little to no treatment options.

\section{Acknowledgments}

Editorial support for this manuscript was provided by Precept Medical Communications (Warren, NJ, USA). Funding for editorial support was provided by Bayer HealthCare (Whippany, NJ, USA).

\section{Disclosure}

Gazala Khan has received compensation from Bayer HealthCare for participation in an advisory board meeting. Rebecca Moss' institution received research support from Bayer HealthCare. Fadi Braiteh is a consultant for Bristol-Myers Squibb, Dendreon, Geonomic Health, and Onyx. He receives compensation from Amgen, Bayer HealthCare, Bristol-Myers Squibb, Caris 4 Life Sciences, Celgene, Genomic Health, INSYS, Myriad, Novartis, and Onyx. Dr Braiteh has also received research funds from Bayer HealthCare, BristolMyers Squibb, Caris 4 Life Sciences, Celgene, Genentech,
Genomic Health, GlaxoSmithKline, Lilly, Merrimack, Myriad, Novartis, Onyx, and Roche. Marc Saltzman has received compensation as a consultant for Bayer HealthCare. He has been on the speakers' bureau for GTX and Archimedes and has been an investigator in multiple pharmaceutical company trials including ASI, Astra Zeneca, Bayer HealthCare, Bristol-Myers Squibb, Boehringer Ingelheim, Celegene, Genetech, Merck, Millennium, and Roche. The authors declare no other conflicts of interest in this work.

\section{References}

1. Fearon ER. Molecular genetics of colorectal cancer. Annu Rev Pathol. 2011;6:479-507.

2. Cancer Genome Atlas Network. Comprehensive molecular characterization of human colon and rectal cancer. Nature. 2012;487(7407):330-337.

3. Kim DD, Eng C. The current state of targeted agents in rectal cancer. Int J Surg Oncol. 2012;2012:406830.

4. National Comprehensive Cancer Networks (NCCN). NCCN Clinical Practice Guidelines in Oncology Colon Cancer. Version 3. Fort Washington, PA: NCCN; 2013.

5. Wilhelm SM, Dumas J, Adnane L, et al. Regorafenib (BAY 73-4506): a new oral multikinase inhibitor of angiogenic, stromal and oncogenic receptor tyrosine kinases with potent preclinical antitumor activity. Int J Cancer. 2011;129(1):245-255.

6. Tsai JH, Lee WM. Tie2 in tumor endothelial signaling and survival: implications for antiangiogenic therapy. Mol Cancer Res. 2009;7(3): 300-310.

7. Mross K, Frost A, Steinbild S, et al. A phase I dose-escalation study of regorafenib (BAY 73-4506), an inhibitor of oncogenic, angiogenic, and stromal kinases, in patients with advanced solid tumors. Clin Cancer Res. 2012;18(9):2658-2667.

8. Strumberg D, Scheulen ME, Schultheis B, et al. Regorafenib (BAY 73-4506) in advanced colorectal cancer: a phase I study. Br J Cancer. 2012;106(11):1722-1727.

9. Eisenhauer EA, Therasse P, Bogaerts J, et al. New response evaluation criteria in solid tumours: revised RECIST guideline (version 1.1). Eur J Cancer. 2009;45(2):228-247.

10. Bayer HealthCare. Patients with metastatic colorectal cancer treated with regorafenib or placebo after failure of standard therapy. In: ClinicalTrials.gov [website on the Internet]. Bethseda, MD: US National Library of Medicine; 2010 [updated July 18, 2013]. Available from: http://clinicaltrial.gov/show/NCT01103323. NLM identifier: NCT01103323. Accessed November 1, 2011.

11. Grothey A, Van Cutsem E, Sobrero A, et al; CORRECT Study Group. Regorafenib monotherapy for previously treated metastatic colorectal cancer (CORRECT): an international, multicentre, randomised, placebocontrolled, phase 3 trial. Lancet. 2013;381(9863):303-312.

12. Stivarga ${ }^{\circledR}$ (regorafenib) tablets, for oral use [prescribing information]. Wayne, NJ: Bayer HealthCare Pharmaceuticals Inc; 2013. Available from: http://labeling.bayerhealthcare.com/html/products/pi/Stivarga PI.pdf. Accessed November 1, 2013.

13. Spruance SL, Reid JE, Grace M, Samore M. Hazard ratio in clinical trials. Antimicrob Agents Chemother. 2004;48(8):2787-2792.

14. Van Cutsem E, Grothey A, Sobrero A, et al. Phase III CORRECT trial of regorafenib in metastatic colorectal cancer ( $\mathrm{mCRC}$ ): overall survival update. Presented at the European Society of Medical Oncology 2012 Congress, September 28-October 2, 2012, Vienna, Austria. Abstract LBA18.

15. Ricotta R, Sartore-Bianchi A, Verrioli A, Vanzulli A, Siena S. Regorafenib for metastatic colorectal cancer. Lancet. 2013;381(9877):1537.

16. Bayer HealthCare Pharmaceuticals Inc. Clinical Study Report 14387 (CORRECT). Wayne, NJ: Bayer HealthCare Pharmaceuticals Inc; 2012: $1-179$. 
17. Grothey A, Van Cutsem E, Sobrero A, et al. Time course of regorafenibassociated adverse events in the phase III CORRECT study. J Clin Oncol. 2012;30(Suppl 34):Abstract 467.

18. Porta C, Paglino C, Imarisio I, Bonomi L. Uncovering Pandora's vase: the growing problem of new toxicities from novel anticancer agents. The case of sorafenib and sunitinib. Clin Exp Med. 2007;7(4):127-134.

19. Lacouture ME, Wu S, Robert C, et al. Evolving strategies for the management of hand-foot skin reaction associated with the multitargeted kinase inhibitors sorafenib and sunitinib. Oncologist. 2008;13(9): 1001-1011.

20. Wood LS, Lemont H, Jatoi A, et al. Practical considerations in the management of hand-foot skin reaction caused by multikinase inhibitors. Community Oncol. 2010;7(1):23-29.

21. Ren Z, Zhu K, Kang H, et al. A randomized controlled phase II study of the prophylactic effect of urea-based cream on the hand-foot skin reaction associated with sorafenib in advanced hepatocellular carcinoma. J Clin Oncol. 2012;30(Suppl):Abstract 4008.

22. Bellmunt J, Eisen T, Fishman M, Quinn D. Experience with sorafenib and adverse event management. Crit Rev Oncol Hematol. 2011;78(1): 24-32.

23. Izzedine H, Ederhy S, Goldwasser F, et al. Management of hypertension in angiogenesis inhibitor-treated patients. Ann Oncol. 2009;20(5): 807-815.

24. Chobanian AV, Bakris GL, Black HR, et al; National Heart, Lung, and Blood Institute Joint National Committee on Prevention, Detection, Evaluation, and Treatment of High Blood Pressure; National High Blood Pressure Education Program Coordinating Committee. The Seventh Report of the Joint National Committee on Prevention, Detection, Evaluation, and Treatment of High Blood Pressure: the JNC 7 report. JAMA. 2003;289(19):2560-2572.

25. Maitland ML, Bakris GL, Black HR, et al; Cardiovascular Toxicities Panel, Convened by the Angiogenesis Task Force of the National Cancer Institute Investigational Drug Steering Committee. Initial assessment, surveillance, and management of blood pressure in patients receiving vascular endothelial growth factor signaling pathway inhibitors. J Natl Cancer Inst. 2010;102(9):596-604.

26. Kollmannsberger C, Soulieres D, Wong R, Scalera A, Gaspo R, Bjarnason G. Sunitinib therapy for metastatic renal cell carcinoma: recommendations for management of side effects. Can Urol Assoc J. 2007;1(Suppl 2):S41-S54.

27. van Geel RM, Beijnen JH, Schellens JH. Concise drug review: pazopanib and axitinib. Oncologist. 2012;17(8):1081-1089.
28. Stein A, Voigt W, Jordan K. Chemotherapy-induced diarrhea: pathophysiology, frequency and guideline-based management. Ther Adv Med Oncol. 2010;2(1):51-63.

29. Benson AB 3rd, Ajani JA, Catalano RB, et al. Recommended guidelines for the treatment of cancer treatment-induced diarrhea. J Clin Oncol. 2004;22(14):2918-2926.

30. Campos MP, Hassan BJ, Riechelmann R, Del Gigilo A. Cancer-related fatigue: a practical review. Ann Oncol. 2011;22(6):1273-1279.

31. Schwandt A, Wood LS, Rini B, Dreicer R. Management of side effects associated with sunitinib therapy for patients with renal cell carcinoma. Onco Targets Ther. 2009;2:51-61.

32. Wood LS. Managing the side effects of sorafenib and sunitinib. Community Oncol. 2006;3:558-562.

33. Nexavar ${ }^{\circledR}$ (sorafenib) tablets, oral [prescribing information]. Wayne, NJ: Bayer HealthCare Pharmaceuticals Inc; 2010. Available from: http://www.accessdata.fda.gov/drugsatfda_docs/ label/2010/021923s008s009lbl.pdf. Accessed November 1, 2013.

34. Sutent ${ }^{\circledR}$ (sunitinib malate) capsules, oral [prescribing information]. New York, NY: Pfizer Inc; 2011. Available from: http:/www. accessdata.fda.gov/drugsatfda_docs/label/2011/021938s13s17s18lbl. pdf. Accessed November 1, 2013.

35. Argiles G, Troiani T, Rivera F, et al. First-line treatment with regorafenib in combination with mFOLFOX6 for metastatic colorectal cancer: a single-arm, open-label phase II clinical trial. Presented at the European Society of Medical Oncology 2013 Congress, September 27-October 1, 2013, Amsterdam, the Netherlands. Abstract 2367

36. UNC Lineberger Comprehensive Cancer Center. Regorafenib+ FOLFIRI Versus Placebo+FOLFIRI as 2nd Line Tx in Metastatic Colorectal Cancer. In: ClinicalTrials.gov [website on the Internet]. Bethseda, MD: US National Library of Medicine; 2011 [updated August 20, 2013]. Available from: http://clinicaltrial.gov/show/ NCT01298570. NLM identifier: NCT01298570. Accessed November 1, 2011.

37. Bayer HealthCare. Regorafenib in subjects with metastatic colorectal cancer (CRC) who have progressed after standard therapy (CONSIGN). In: ClinicalTrials.gov [website on the Internet]. Bethseda, MD: US National Library of Medicine; 2012 [updated October 23, 2013]. Available from: http://clinicaltrial.gov/show/NCT01538680. NLM identifier: NCT01538680. Accessed November 1, 2011.
Cancer Management and Research

\section{Publish your work in this journal}

Cancer Management and Research is an international, peer-reviewed open access journal focusing on cancer research and the optimal use of preventative and integrated treatment interventions to achieve improved outcomes, enhanced survival and quality of life for the cancer patient The journal welcomes original research, clinical \& epidemiological

\section{Dovepress}

studies, reviews \& evaluations, guidelines, expert opinion \& commentary, case reports \& extended reports. The manuscript management system is completely online and includes a very quick and fair peerreview system, which is all easy to use. Visit http://www.dovepress.com/ testimonials.php to read real quotes from published authors. 